\title{
Evaluation of a Direct Oral Anticoagulant Stewardship Program: Analysis of a Drug Consult Review Process and Population-Based Management Tool
}

\author{
Haley A Henry ${ }^{1}$, Carli C Smith ${ }^{1}$, Bishoy Ragheb ${ }^{1}$, Ashley M Thomas ${ }^{1}$ and Jennifer W Baker ${ }^{1 *}$ \\ ${ }^{1}$ Veteran Affairs Tennessee Valley Healthcare System, Nashville, TN \\ *Corresponding Author: Jennifer W Baker, Veteran Affairs Tennessee Valley Healthcare System, Nashville, TN; E-mail: Jennifer.Baker4@va.gov
}

Received: October 23, 2019; Accepted: November 13; Published: November 22, 2019

\begin{abstract}
Background: With the approval of Direct Oral Anticoagulants (DOAC), anticoagulation management has been transformed in both stroke prevention and venous thromboembolism prophylaxis and treatment. Despite evidence-based dosing guidance there has been large variation in prescribing practices that may lead to negative outcomes. The purpose of this two-part study is to evaluate the impact of a DOAC stewardship program on appropriate DOAC prescribing and use.
\end{abstract}

Methods: Patients were included in part one of the study if they were prescribed initial DOAC therapy from October 15, 2017 - October 15, 2018 with either a general or DOAC specific drug consult. A manual chart review was then conducted for data points including: anticoagulation indication, DOAC dose, serum creatinine, weight, age, consult approval or denial, and reasons for denial. Part two of the study included patients identified through a VA Population-Based Management Tool (PBMT) from January 1, 2018 - September 30, 2018. A manual chart review was then conducted for data points including: flag category, interventions made, and interventions accepted. Patients were excluded in both arms of the study if the duration of DOAC therapy was less than 20 days, incomplete chart review, or if DOAC therapy was prescribed by a non-VA provider.

Results: A total of 592 consults were included in the final analysis in part one of the study. Of the 233 general consults evaluated, 212 (91.0\%) were deemed appropriate, $15(6.4 \%)$ inappropriate, and $6(2.6 \%)$ as clinical grey areas. Of the 233 DOAC specific drug consults evaluated, $218(93.6 \%)$ were deemed appropriate, $1(0.4 \%)$ inappropriate, and $14(6.0 \%)$ as clinical grey areas. There was a significant difference in consults worked inappropriately $(\mathrm{p}=0.0004)$. A total of 317 PBMT interventions were included in the final analysis in part two of the study. Of those interventions that were actively acknowledged, 233 (95.9\%) interventions were completed.

Conclusion: Implementation of a DOAC stewardship program in a healthcare system promotes appropriate and optimal use as well as safety monitoring of DOACs. A drug-specific consult review process improves inappropriate approval or denial of DOAC therapy while the utilization of a populationbased management tool efficiently identifies critically important interventions necessary to ensure safe and appropriate use of DOACs.

\section{Introduction}

Two disease states that often require anticoagulation for either prophylaxis or treatment are nonvalvular atrial fibrillation (AF) and venous thromboembolism (VTE). Until recently, the primary option for oral anticoagulation was warfarin, a vitamin K antagonist. In 2010, the first Direct Oral Anticoagulant (DOAC), dabigatran, was FDA approved and since then, other DOACs have received FDA approval within the United States (rivaroxaban, apixaban and edoxaban) [1-4]. With the approval of these agents, anticoagulation management has been transformed in both stroke prevention and VTE prophylaxis and treatment. DOACs require no routine lab monitoring to assess anticoagulation effect due to their favorable pharmacokinetic and pharmacodynamic profile when compared with warfarin. However, DOACs are still associated with serious bleeding risks and possess characteristics different from warfarin, including: renal elimination, short duration of action, different drug-drug interactions, administration considerations, and dosing based off of appropriate indication.
Despite evidence-based dosing guidance, there has been large variation in prescribing practices that may lead to negative outcomes. Steinberg and colleagues were the first to analyze the association between DOAC doses and clinical outcomes in patients with nonvalvular AF. Their study, through the ORBIT-AF II Registry, concluded that off-label doses of DOAC therapy for prevention of stroke in nonvalvular AF are associated with increased risk for adverse events [5]. In addition to prescribing practices, adherence to anticoagulants effects patient outcomes. Shore, et al assessed the adherence component of DOAC therapy by specifically studying patients on dabigatran in Veterans Affairs (VA) hospitals. Their study found that one-quarter of patients demonstrated sub-optimal adherence to dabigatran and poor adherence was associated with an increased risk for stroke and all-cause mortality [6]. Dreijer and colleagues determined $8.3 \%$ of medication errors from the hospital and primary care settings were caused from anticoagulation agents, with most error reports concerning the prescribing phase of the medication process [7]. With these results and common practices 
today, it is imperative that prescribers are aware of the need for oversight of DOAC prescribing. Additionally, studies have shown that a pharmacist driven monitoring program improves appropriate prescribing and monitoring of DOACs used for FDA approved indications. Miele, et al. revealed that appropriate prescribing of DOAC therapy was improved after implementing a pharmacist driven monitoring program through a pre- and post-intervention study. They found that $32.4 \%$ of doses administered in the pre-intervention group were considered inappropriate, compared to $13.8 \%$ in the postintervention group. Appropriate prescribing included FDA approved indications and appropriate doses of DOAC therapy based on renal function [8].

Tennessee Valley Healthcare System (TVHS) has pioneered a DOAC stewardship program that includes a DOAC drug specific consult to ensure appropriate prescribing at initiation and then long-term surveillance of patients on DOAC therapy by an ongoing population-based management tool. The goal of this stewardship program is to improve appropriate DOAC utilizations by streamlining the initial DOAC consult process along with ongoing management by utilizing anticoagulation clinical pharmacy specialists (ACC CPS) and a DOAC population-based management tool (PBMT). Previously, DOAC therapy was approved through a generic drug consult review process evaluated by a general clinical pharmacy specialist (CPS). This created a lack of consistency between DOAC approval on a patient to patient basis. The newly developed drug consult review process utilizes both a DOAC drug specific consult along with an ACC CPS. TVHS hopes to improve DOAC utilization with regards to appropriate indication, dosing, and safety through a more specific format of DOAC approval. The DOAC PBMT is used within the VA system to allow for continuous review of safety and compliance parameters for optimal care of patients who are prescribed DOAC therapy. The management tool identifies eight flags including: appropriate dosing, valve replacement, notable labs, overdue labs, critical drug interactions, active Non-Steroidal Inflammatory Drug (NSAID) use, overdue refill greater than 4 weeks and renewal due in next 30 days. It is the responsibility of the ACC CPS to actively review the PBMT daily. Upon review, if a flag is present, the ACC CPS will document their assessment in the patient's electronic medical record; therefore, notifying the provider of a recommendation or change regarding the patient's current DOAC therapy. In this study, we will evaluate the impact of a DOAC stewardship program on DOAC utilization at TVHS.

\section{Methods}

This multi-site, single center, retrospective cohort study was conducted at TVHS, which includes sites in Nashville and Murfreesboro, TN, as well as patients enrolled at any of the healthcare system's 13 Community Based Outpatient Clinics (CBOCs) throughout Middle Tennessee, southern Kentucky, and northern Georgia.

Patients were included in part one of the study if they had an active DOAC prescription from October 15, 2017 to October 15, 2018 and 18 years of age or greater. Patients were excluded if the prescription was prescribed for short term therapy (inpatient use only and traveling veterans), written by orthopedics, there was an incomplete data set, or if a consult was denied based on criteria for use (CFU). Patients were included in part two of the study if they had an active DOAC prescription from January 1, 2018 to September 30, 2018 with a note title "Anticoagulation Eval and Mgt Secure Messaging" in the electronic medical record indicating an ACC CPS intervention and 18 years of age or greater. Patients were excluded if the prescription was prescribed for short term therapy (inpatient use only and traveling veterans), if the note title was used in error or the patient relocated to a different VA system. The primary endpoint of this study is to determine the appropriate use of DOAC therapy through a drug consult review process. The secondary endpoints are to determine how the drug consult review process and the utilization of a population-based management tool influences safety outcomes of initial and ongoing DOAC therapy including, concomitant antiplatelet therapy in part one and the PBMT flag categories in part two.

Patients who were prescribed direct oral anticoagulation therapy from October 15, 2017 - October 15, 2018 were identified through data warehouse extraction. A manual chart review was conducted for data points including: anticoagulation indication, DOAC dose at time of consult submission, initial or renewal consult, documented labs, documented weight, consult approval or denial, and rational for approval or denial. Evaluated consults were then categorized as appropriate, inappropriate, or clinical grey areas (Appendix 1). Patients followed by the PBMT from March 24, 2018 to September 24, 2018 were identified through data warehouse extraction using the note title "Anticoagulation Eval \& Mgt Secure Messaging." A manual chart review was conducted for data points including the PBMT flag categories (Appendix 2), interventions actively acknowledged and interventions completed. The sample size was calculated based on the primary objective to determine appropriate use of DOAC therapy through a drug consult review process. The sample included all patients who meet study criteria that received DOAC therapy during the pre-determined timeframe. We wanted to see at least a $15 \%$ difference regarding the number of appropriate approvals/denials between the generalized and the specialized consult review process. Using these two data points, the effect size was found to be $15 \%$ with alpha set at 0.05 and beta set at 0.20 . The study required 133 patients in each arm to be adequately powered. Chi square and fisher exact were used to calculate the $\mathrm{p}$-value for the categorical data presented.

\section{Results}

Table 1 displays the baseline demographics and clinical characteristics of the two cohorts. The two groups did not different significantly with respect to age, gender, and race. However, there was a statistically significant difference found in use of rivaroxaban and dabigatran between the two cohorts. The authors attribute this difference to the delineated questions of the drug-specific consult. (Table 1)

\section{Part I: Drug consult review process}

\section{Primary Outcomes}

Two thousand twenty-one unique patients were extracted from October 15, 2017 - October 15, 2018. Due to time constraints and sample size being met, after the initial data pull the date range was 
Jennifer W Baker (2019) Evaluation of a Direct Oral Anticoagulant Stewardship Program: Analysis of a Drug Consult Review Process and PopulationBased Management Tool

shortened to January 13, 2018 to July 13, 2018. All consults in the generalized cohort were evaluated $(\mathrm{n}=296)$ and consults in the specialized cohort were randomized to meet a matching sample size $(n=296)$; therefore, a total of 592 DOAC consults were evaluated. In the generalized cohort, 233 consults were included for study evaluation with 63 excluded for various reasons (incomplete data set, orthopedic patients, denial based on CFU). In the specialized cohort, 233 consults for study evaluation with 63 excluded for various reasons (short term therapy, incomplete data set, orthopedic patient, denial based on $\mathrm{CFU})$. Table 2 illustrates the number of evaluated consults deemed appropriate, inappropriate, and those classified as clinical grey areas. Of the 233 general consults worked, 212 were deemed appropriate, 15 inappropriate, and 6 as clinical grey areas. Of the 233 DOAC Specific Drug consults worked, 218 were deemed appropriate, 1 inappropriate, and 14 as clinical grey areas. Statistical significance was seen only in those consults worked inappropriately. (Table 2)

Table 1. Baseline characteristics for the consult review process

\begin{tabular}{|c|c|c|c|}
\hline & $\begin{array}{c}\text { Generalized Consult } \\
n=233(\%)\end{array}$ & $\begin{array}{c}\text { DOAC Specific Consult } \\
\mathbf{n}=\mathbf{2 3 3}(\%)\end{array}$ & P-value \\
\hline \multicolumn{4}{|l|}{ Age } \\
\hline Average $\pm \mathrm{SD}$ & $70.9 \pm 11$ & $69.9 \pm 12$ & 0.3489 \\
\hline Male sex - no. (\%) & $231(99.1)$ & $227(97.4)$ & 0.2848 \\
\hline \multicolumn{4}{|l|}{ Race or ethnic group - no. (\%) } \\
\hline White & $194(83.3)$ & $204(87.5)$ & 0.2375 \\
\hline Black or African American & $20(8.6)$ & $17(7.3)$ & 0.7323 \\
\hline Asian & 0 & $1(<1)$ & 1.0000 \\
\hline Native Hawaiian or Pacific Islander & 0 & $0(0)$ & 1.0000 \\
\hline American Indian or Alaska Native & $1(<1)$ & $1(<1)$ & 1.0000 \\
\hline Unknown & $18(7.7)$ & $10(4.3)$ & 0.1715 \\
\hline \multicolumn{4}{|c|}{ Direct Oral Anticoagulant (DOAC) - no (\%) } \\
\hline Apixaban & $85(36.5)$ & $82(35.2)$ & 0.8468 \\
\hline Rivaroxaban & $56(24)$ & $78(33.5)$ & 0.0316 \\
\hline Dabigatran & $91(39.1)$ & $65(27.9)$ & 0.0141 \\
\hline Edoxaban & $1(<1)$ & $3(1.3)$ & 0.6156 \\
\hline No preference & $0(0)$ & $5(2.1)$ & 0.0721 \\
\hline \multicolumn{4}{|l|}{ Indication for Use } \\
\hline Nonvalvular atrial fibrillation & $162(69.5)$ & $177(76)$ & 0.1452 \\
\hline Venous Thromboembolism (VTE) & $64(27.5)$ & $46(19.7)$ & 0.0637 \\
\hline Other & $7(3)$ & $10(4.3)$ & 0.6212 \\
\hline
\end{tabular}

*Indication for Use - "other" includes non-FDA approved indications

Table 2. Categorization of consult approval or denial

\begin{tabular}{|c|c|c|c|}
\hline Process & Appropriate (\%) & Inappropriate (\%) & Clinical Grey Area (\%) \\
\hline Generalized (n=233) & $212(91)$ & $15(6.4)$ & $6(2.6)$ \\
\hline Specialized (n=233) & $218(93.6)$ & $1(0.4)$ & 0.1075 \\
\hline P values & 0.3859 & $\mathbf{0 . 0 0 0 4}$ & 145 \\
\hline
\end{tabular}


Jennifer W Baker (2019) Evaluation of a Direct Oral Anticoagulant Stewardship Program: Analysis of a Drug Consult Review Process and PopulationBased Management Tool

Of the 15 consults worked inappropriately in the generalized process, inappropriate interventions identified included: non-FDA approved dosing, critical drug-drug interactions, non-FDA approved indications, no indication provided, and hepatic dysfunction. The one consult worked inappropriately in the specialized process, the inappropriate intervention identified was non-FDA approved dosing.

\section{Secondary Outcomes}

There were no interventions made in the generalized consult review process regarding concomitant antiplatelet use, however there were 29 recommendations made to either discontinue the P2Y12 inhibitor or discontinue/decrease the dose of aspirin in the DOAC specific consult process. Of the 29 recommendation that were made, $13(45 \%)$ recommendations were completed, specifically 9 (69\%) were discontinued and 4 (31\%) doses were decreased.

\section{Part II: Population Based Management Tool}

One thousand fourteen notes with the title "Anticoagulation Eval and Mgt Secure Messaging" were extracted from March 24, 2018 to September 24, 2018 and randomized using Microsoft excel. Three hundred four notes were evaluated to collect the pertinent information as noted above in the "data collection" section. Two-hundred ninetytwo notes were included as 12 notes were excluded for various reasons (orthopedic patient, use of wrong note title, relocation to another VA and no active DOAC prescription). Of the 292 notes that were evaluated, 267 notes only included interventions made on one flag, where as 25 notes included interventions on 2 flags resulting in a total of 317 interventions made.

Table 3 displays the number of interventions made regarding the flag category specified by the population-based management tool. By far, the intervention that was made most commonly was alerting the provider that the prescription needed to be renewed (30\%), followed by overdue refill > 4 weeks (22\%), overdue labs $(15 \%)$ and inappropriate dosing (11\%). From the 317 interventions that were made 242 (76.7\%) interventions were actively acknowledged. Of those that were actively acknowledged, 233 (95.9\%) interventions were completed (meaning a medication was renewed, refilled, labs were ordered, medications changed, etc.) with specific percentages regarding each flag noted in table 4. (Table 3, Table 4)

\section{Discussion}

As the anticoagulation paradigm begins to shift from warfarin to DOAC therapy, the use of these medications should still be managed and monitored carefully to prevent unwanted harm. To our knowledge, this is the one of the first studies analyzing the implementation of an ACC CPS run DOAC stewardship program that includes a drug-specific consult and a continuous PBMT evaluated by ACC CPS. When using a generalized consult review process, more consults were deemed to be evaluated inappropriately compared to a specialized consult review process. The generalized process included a consult with minimal requirements for completion (drug, dose, and reason why patient was not candidate for preferred formulary agent, dabigatran) and was then reviewed by a general clinical pharmacy specialist. With the implementation of a DOAC stewardship program, a drug-specific consult was created and is now reviewed by an ACC CPS. For completion, the drug-specific consult must include: drug, dose, indication (as well as details associated with indication), appropriate baseline labs (Hgb/Hct, SCr, AST/ALT), bleeding history, antiplatelet use, NSAID use, and use of pillbox.

Table 3. Interventions made via the population-based management tool

\begin{tabular}{|l|c|}
\hline & $\begin{array}{c}\text { Interventions made } \\
\mathbf{n}=\mathbf{3 1 7}(\%)\end{array}$ \\
\hline Overdue labs & $49(15)$ \\
\hline Notable labs & $19(6)$ \\
\hline Active NSAID use & $35(11)$ \\
\hline Overdue refill $>$ 4 weeks & $69(22)$ \\
\hline Dosing & $39(12)$ \\
\hline Critical drug-drug interactions & $11(3)$ \\
\hline Valve replacement & $1(0.33)$ \\
\hline Renewal due & $95(30)$ \\
\hline P2Y12i use & $2(0.66)$ \\
\hline
\end{tabular}

Table 4. Interventions actively acknowledged and completed

\begin{tabular}{|c|c|c|c|}
\hline & $\begin{array}{l}\text { Interventions } \\
\text { actively } \\
\text { acknowledged } \\
\mathbf{n}=\mathbf{2 4 3}\end{array}$ & $\begin{array}{l}\text { Interventions } \\
\text { completed n } \\
\quad=233\end{array}$ & $\begin{array}{c}\text { Percent } \\
\text { completed } \\
\text { based on } \\
\text { those actively } \\
\text { acknowledge }\end{array}$ \\
\hline Overdue labs & 28 & 27 & $96.4 \%$ \\
\hline Notable labs & 12 & 10 & $83.3 \%$ \\
\hline Active NSAID use & 21 & 16 & $76.1 \%$ \\
\hline $\begin{array}{c}\text { Overdue refill > } \\
\text { weeks }\end{array}$ & 53 & 52 & $98.1 \%$ \\
\hline Dosing & 31 & 24 & $77.4 \%$ \\
\hline $\begin{array}{l}\text { Critical drug-drug } \\
\text { interactions }\end{array}$ & 9 & 7 & $77.8 \%$ \\
\hline Valve replacement & 1 & 0 & $0 \%$ \\
\hline Renewal due & 87 & 87 & $100 \%$ \\
\hline P2Y12i use & 1 & 0 & $0 \%$ \\
\hline
\end{tabular}

The results of this study are consistent with previous studies finding that implementing a pharmacist driven monitoring program reduced inappropriate prescribing of DOAC therapy in adult patients with an indication for nonvalvular AF and/or VTE prophylaxis and treatment [8]. In our study, the following inappropriate uses of DOAC therapy were identified: non-FDA approved indications (apical thrombus, cryptogenic stroke), critical drug-drug interaction (strong CYP3A4 and P-glycoprotein inducers), non-FDA approved dosing (lead-in dosing provided for AF, subtherapeutic use of apixaban, use of 
apixaban with $\mathrm{CrCl}<25 \mathrm{~mL} / \mathrm{min}$ ), and the use of dabigatran in hepatic dysfunction. ACC CPS were able to use the consult process to make key interventions by recommending a more clinically appropriate DOAC for scenarios including: use of apixaban due to age $>75$ years old or declining renal function, denial of dabigatran due to use of pillbox, and use of rivaroxaban for ease of compliance. By utilizing the PBMT, our study illustrates that continuous monitoring of DOAC therapy is necessary as patient's clinical status has the potential to change while on DOAC therapy. The authors attribute the one-fourth of interventions not actively acknowledge as due to the lack of education provided to prescribers prior to the implementation of the DOAC stewardship program. However, almost $96 \%$ of the interventions that were actively acknowledge were completed, showing the importance of additional surveillance methods rather than relying on prescriber chart reviews. Moving forward, the authors plan to provide educational sessions to prescribers regarding the importance of the PBMT hoping to eliminate alert fatigue and engage providers in the ongoing efforts of the DOAC stewardship program.

There are a few limitations of this study to consider. First, when categorizing the evaluated consults, clinical grey areas have the potential to differ depending on the consult reviewer's clinical interpretation of current literature. Second, throughout data collection, four reviewers were trained based on the derived protocol; however, due to the abundant number of unique patients and charts to be reviewed data could have been assessed differently between reviewers. Impacts of this study support the need for a DOAC stewardship program in a healthcare system to promote appropriate and optimal use as well as safety monitoring of DOACs. A drug-specific consult review process improves inappropriate approval or denial of DOAC therapy while the utilization of a PBMT efficiently identifies critically important interventions necessary to ensure safe and appropriate use of DOACs.

\section{Appendix 1: Clinical Grey Areas}

- Use of apixaban due to age $>75$ years old

- Denial of dabigatran due to use of pillbox

- LV Thrombus (if failed or cannot take warfarin)

- Weight $(>120 \mathrm{~kg})$

- Labs not collected within 90 days

- Use of DOAC versus heparin or enoxaparin for lead-in for treatment of VTE

- Non-FDA approved use of DOACs per Landmark Clinical Trials

- $\mathrm{CrCl} 15-25 \mathrm{~mL} / \mathrm{min}$ for use of apixaban in A. Fib

- $\mathrm{CrCl} 15-30 \mathrm{~mL} / \mathrm{min}$ for use of rivaroxaban in A. Fib

\section{Appendix 2: Population-Based Management Tool (PBMT) Flags}

- Overdue labs

- Results include all patients where either their most recent hemoglobin OR most recent platelet OR most recent serum creatinine is overdue per patient's monitoring frequency
- Monitoring frequency defaults to 12 months for all labs except 6 months for serum creatinine for patients that are 75 years of age or more and/or have a $\mathrm{CrCl}$ of less than $60 \mathrm{~mL} / \mathrm{min}$

- Once the overdue lab(s) are resulted (once daily in the morning) the patient will no longer appear in this column. In addition, after review of the patient, monitoring frequency (for serum creatinine) can be reset to 2 weeks, 1, 3, 6 or 12 months as determined clinically appropriate

- Notable labs

- Most recent platelets $<100 \times 10^{9} / \mathrm{L}$ if the second most recent value was above $100 \times 10^{9} / \mathrm{L} \mathrm{OR}$ any platelet value $<50 \times 10^{9}$ $/ \mathrm{L}$ OR hemoglobin $<10 \mathrm{~g} / \mathrm{dL}$ or AST/ALT $>135 / 120 \mathrm{U} / \mathrm{L}$ OR a hemoglobin drop $>2 \mathrm{~g} / \mathrm{dL}$ since previous hemoglobin with resultant value $<13.1 \mathrm{~g} / \mathrm{dL}$ for males and $<11.0 \mathrm{~g} / \mathrm{dL}$ for females

- Active NSAID use

- Patients with an active non-aspirin NSAID based on VA Drug Class MS101 and MS102

- Active includes prescriptions with status 'ACTIVE', 'SUSPENDED', 'PROVIDER HOLD', 'HOLD', 'PENDING'

- This will include non-VA prescriptions

- Valve replacement

- Results include all patients with an ICD code for a prosthetic (bioprosthetic or mechanical) heart valve on their problem list, attached to two outpatient visits within the last 2 years, or as inpatient discharge diagnosis within the last two years

- Dosing flag

- Results include all patients whose renal function (by CockcroftGault with actual body weight) falls above or below specified cutoffs based on agent, indication and if applicable selected drug interactions as per FDA approved package inserts

- Results also include patients whose dose does not appear to match indication and duration or other non-renal dose modifying characteristics (e.g. high dose apixaban for PE/ DVT > 6 months, low dose apixaban for PE/DVT within first 6 months, dose/indication mismatch based on age and body weight)

- Overdue refill $>4$ weeks

- Results include patients for whom it has been more than 4 weeks since their day supply would be expected to run out (based on released date plus one additional week to allow for shipment)

- Renewal due in next 30 days

- Patients for whom the ordered days supply is scheduled to expire within the next 30 days

- P2Y12i use

- Antiplatelet therapy includes aspirin, clopidogrel (Plavix ${ }^{\circledR}$ ), ticagrelor (Brilinta ${ }^{\circledR}$ ), prasugrel (Effient ${ }^{\circledR}$ ) 
- Critical drug-drug interactions

\begin{tabular}{|l|l|}
\hline DOAC & Drug Interactions \\
\hline Dabigatran & $\begin{array}{l}\text { Flag if active warfarin, apixaban, edoxaban, } \\
\text { rivaroxaban, rifampin, primidone, St. John's } \\
\text { Wort, phenobarbital, carbamazepime, } \\
\text { phenytoin, dronedarone, cyclosporine, } \\
\text { tacrolimus, itraconazole, ketoconazole, } \\
\text { pasaconazole, voriconazole, rifampicin, } \\
\text { saquinavir, ritonavir, indinavir, nelfinavir, } \\
\text { amprenavir, lopinavir, fosamprenavir, } \\
\text { atazanavir, tipranavir or darunavir }\end{array}$ \\
\hline Rivaroxaban & $\begin{array}{l}\text { Flag if active warfarin, apixaban, } \\
\text { edoxaban, dabigatran, rifampin, primidone, } \\
\text { phenobarbital, carbamazepime, phenytoin, } \\
\text { itraconazole, ketoconazole, pasaconazole, } \\
\text { voriconazole, rifampicin, saquinavir, ritonavir, } \\
\text { indinavir, nelfinavir, amprenavir, lopinavir, } \\
\text { fosamprenavir, atazanavir, tipranavir, darunavir } \\
\text { or St. John's Wort }\end{array}$ \\
\hline Epixaban & $\begin{array}{l}\text { Flag if active warfarin, apixaban, rivaroxaban, } \\
\text { dabigatran, rifampin, primidone, phenobarbital, } \\
\text { carbamazepime, phenytoin, rifampicin, } \\
\text { saquinavir, ritonavir, indinavir, nelfinavir, } \\
\text { amprenavir, lopinavir, fosamprenavir, } \\
\text { atazanavir, tipranavir, darunavir or St. John's } \\
\text { Wort }\end{array}$ \\
\hline $\begin{array}{l}\text { Flag if active warfarin, rivaroxaban, } \\
\text { edoxaban, dabigatran, rifampin, primidone, } \\
\text { phenobarbital, carbamazepime, phenytoin, } \\
\text { itraconazole, ketoconazole, pasaconazole, } \\
\text { voriconazole, rifampicin, saquinavir, ritonavir, } \\
\text { indinavir, nelfinavir, amprenavir, lopinavir, } \\
\text { fosamprenavir, atazanavir, tipranavir, darunavir } \\
\text { or St. John's Wort }\end{array}$ \\
\hline
\end{tabular}

- Other critical drug interactions

- Active cancer pharmacotherapy

- Flag if: active VA cancer pharmacotherapy in a patient with diagnosis of DVT/PE \pm atrial fibrillation or atrial flutter

- Active includes prescriptions with status 'ACTIVE', 'SUSPENDED', 'PROVIDER HOLD', 'HOLD', 'PENDING' for any of the interacting drugs or cancer pharmacotherapy

- Non-VA medications will be included in the analysis for critical drug-drug interactions. Patients with non-VA DOAC prescriptions will not be identified by this tool

\section{References}

1. Eliquis [package insert]. New York, NY: Pfizer Pharmaceuticals, Inc; 2012.

2. Xarelto [package insert]. Titusville, NJ: Janssen Pharmaceuticals, Inc; 2011.

3. Pradaxa [package insert]. Ridgefield, CT. Boehringer Ingelheim Pharmaceuticals, Inc; 2011.

4. Savaysa [package insert]. Tokyo, Japan. Daiichi Sankyo Company, Limited; 2017.

5. Steinberg B, Shrader P, Thomas L, Ansell J, Fonarow GC, et al. (2016) Off-label dosing of non-vitamin $\mathrm{K}$ antagonist oral anticoagulants and adverse outcomes, The ORBIT-AF II Registry. JACC 68: 2597-2604.

6. Shore S, Carey E, Turakhia M, Jackevicius CA, Cunningham F, et al. (2014) Aherence to dabigatran therapy and longitudinal patient outcomes: insights from the Veterans Health Administration. Am Heart J 167: 810-817.
7. Dreijer AR, Diepstraten J, Bukkems VE, Mol PGM, Leebeek FWG, et al. (2019) Anticoagulant medication errors in hospitals and primary care: a cross-sectional study. Int J Qual Health Care 31: 346-352.

8. Miele C, Taylor M, Shah A (2017) Assessment of direct oral anticoagulant prescribing and monitoring pre- and post-implementation of a pharmacy protocol at a community teaching hospital. Hosp Pharm 52: 207-213.

\section{Citation:}

Haley AH, Carli CS, Bishoy R, Ashley MT and Jennifer WB (2019) Evaluation of a Direct Oral Anticoagulant Stewardship Program: Analysis of a Drug Consult Review Process and Population-Based Management Tool. J Cardiol Clin Pract, Volume 2(2): 1-6. 